\section{Infections After Receipt of Bacterially Contaminated Umbilical Cord Blood-Derived Stem Cell Products for Other Than Hematopoietic or Immunologic Reconstitution — United States, 2018}

Kiran M. Perkins, $\mathrm{MD}^{1}$; Samantha Spoto, $\mathrm{MSPH}^{2}$; Danielle A. Rankin, $\mathrm{MPH}^{2}$; Nychie Q. Dotson, $\mathrm{MPH}^{2}$; Mary Malarkey ${ }^{3}$; Melissa Mendoza, $\mathrm{JD}^{3}$; Lorrie McNeill ${ }^{3}$; Paige Gable ${ }^{1}$; Krista M. Powell, MD ${ }^{1}$

The only Food and Drug Administration (FDA)-approved stem cell products are derived from umbilical cord blood, and their only approved use is hematopoietic and immunologic reconstitution (1). On September 17, 2018, the Texas Department of State Health Services received notification of Enterobacter cloacae and Citrobacter freundii bloodstream infections in three patients who had received injections or infusions of non-FDA-approved umbilical cord blood-derived stem cell products processed by Genetech, Inc., and distributed by Liveyon, LLC, for other than hematopoietic or immunologic reconstitution at an outpatient clinic on September 12. Patient isolates of $E$. cloacae had identical pulsed-field gel electrophoresis patterns, suggesting a common source. On September 22, the Florida Department of Health received notification of Escherichia coli, Enterococcus faecalis, and Proteus mirabilis joint infections in four patients who had received injections of these same products at an orthopedic clinic during February 15August 30, 2018, also for other than hematopoietic or immunologic reconstitution. Cultures of unopened products from the clinic by a Florida hospital identified contamination with E. coli and E. faecalis. In response, on September 28, Liveyon issued a voluntary recall and immediately discontinued purchase of the Genetech-processed stem cell products $(2,3)$. On October 4, CDC issued a nationwide call for reports of culture-confirmed infections in patients who had received the Liveyon product.

As of December 14, CDC has received reports of infections in 12 patients from three states, including the initial Florida and Texas cases: Texas (seven), Florida (four), and Arizona (one). Infection types included bloodstream infections, joint infections, and epidural abscesses, among others. All 12 patients received infusions or injections of Liveyon's product before the recall. Among 11 patients for whom conditions prompting product administration were known, all had nonhematopoietic conditions such as pain or orthopedic conditions. All patients were hospitalized; none died (Table).

CDC tested unopened vials obtained from the Texas and Florida clinics where the initial patients had received the product. The six vials from Texas had the same cord-blood donor and processing date as those that had been administered to the patients with infections. E. cloacae was isolated from all six vials; $C$. freundii also was isolated from five. The four vials from Florida were from different donors and processing dates than were the vials from Texas. E. coli was isolated from one of two vials from the same cord-blood donor and processing date; E.coli and E. faecalis were isolated from one of two vials from two unique donors with unique processing dates.

Ongoing investigations include active case finding, additional laboratory testing to compare clinical and product isolates, onsite assessments of health care facility infection control and injection safety practices, and investigation of manufacturing practices (including distribution); initial investigation suggests that bacterial contamination occurred before distribution. Umbilical cord blood cannot be decontaminated after collection because there are currently no validated processes for sterilization, so manufacture of derived products must be highly controlled to prevent distribution of contaminated products (4). The Genetech-processed, Liveyon-distributed product is not FDAapproved or lawfully marketed. Though Genetech and Liveyon are registered with FDA, such registration is not a form of FDA approval. FDA registration alone does not demonstrate compliance of firms or their products with the law.

Regardless of when contamination occurred, this investigation highlights the serious potential risks to patients of stem cell therapies administered for unapproved and unproven uses other than hematopoietic or immunologic reconstitution (5). Although the safety and efficacy of stem cells for other than hematopoietic or immunologic reconstitution have not been well established $(1,4)$, many companies, clinics, and clinicians continue to market products from various sources as treatment for orthopedic, neurologic, and rheumatologic conditions without FDA approval. Such clinics and providers operate in outpatient settings, which often have less robust oversight of infection control measures, including injection safety and medication preparation ( 6 ), potentially amplifying risk to patients. Therefore, FDA has recommended that patients avoid receiving such products outside controlled clinical studies being conducted under an investigational new drug application; these settings help ensure that appropriate manufacturing and safety reporting procedures are followed (1). Health care professionals and consumers should report any adverse events related to treatment with the Genetech/Liveyon products or any unapproved stem cell therapies to FDA's MedWatch Safety Information and Adverse Event Reporting Program (https:// www.fda.gov/Safety/MedWatch/). 
TABLE. Clinical characteristics of patients with culture-confirmed infections after receiving umbilical cord blood-derived stem cell products for other than hematopoietic or immunologic reconstitution — United States, 2018

\begin{tabular}{|c|c|c|c|c|c|c|c|c|}
\hline Patient & $\begin{array}{c}\text { Route/Site } \\
\text { of administration }\end{array}$ & $\begin{array}{c}\text { Date } \\
\text { administered }\end{array}$ & Setting & $\begin{array}{l}\text { Condition } \\
\text { prompting product } \\
\text { administration* }\end{array}$ & $\begin{array}{l}\text { Specimen } \\
\text { collection date, } \\
\text { first positive } \\
\text { culture }\end{array}$ & $\begin{array}{l}\text { Organism } \\
\text { isolated }\end{array}$ & Infection site & $\begin{array}{l}\text { Days of initial } \\
\text { hospitalization to } \\
\text { treat infection }\end{array}$ \\
\hline 1 & $\begin{array}{l}\text { Intra-articular injection, } \\
\text { knee and shoulder }\end{array}$ & Feb 15, 2018 & $\begin{array}{l}\text { Orthopedic } \\
\text { clinic }\end{array}$ & $\begin{array}{l}\text { Degenerative joint } \\
\text { disease }\end{array}$ & Feb 21, 2018 & $\begin{array}{l}\text { Escherichia coli, } \\
\text { Proteus mirabilis }\end{array}$ & Knee & 15 \\
\hline 2 & $\begin{array}{l}\text { Intra-articular injection, } \\
\text { lumbar spine }\end{array}$ & Jun 13, 2018 & Pain clinic & Pain & Jun 14, 2018 & Escherichia coli & Bloodstream & 4 \\
\hline 3 & $\begin{array}{l}\text { Intra-articular injection, } \\
\text { lumbar spine }\end{array}$ & Jul 27, 2018 & $\begin{array}{l}\text { Ambulatory } \\
\text { surgery } \\
\text { center }\end{array}$ & Pain & Aug 1, 2018 & $\begin{array}{c}\text { Escherichia coli, } \\
\text { Enterococcus } \\
\text { faecalis }\end{array}$ & $\begin{array}{l}\text { Bloodstream, } \\
\text { lumbosacral } \\
\text { epidural abscess, } \\
\text { discitis, and } \\
\text { vertebral } \\
\text { osteomyelitis }^{\dagger}\end{array}$ & 58 \\
\hline 4 & $\begin{array}{l}\text { Intra-articular injection, } \\
\text { knee and shoulder }\end{array}$ & Aug 3, 2018 & $\begin{array}{l}\text { Orthopedic } \\
\text { clinic }\end{array}$ & Unknown & Aug 10, 2018 & $\begin{array}{c}\text { Escherichia coli, } \\
\text { Enterococcus } \\
\text { faecalis }\end{array}$ & Knee & 30 \\
\hline 5 & $\begin{array}{l}\text { Intra-articular injection, } \\
\text { shoulders }\end{array}$ & Aug 14, 2018 & $\begin{array}{l}\text { Chiropractic } \\
\text { clinic }\end{array}$ & Osteoarthritis & Aug 29, 2018 & Escherichia coli & $\begin{array}{l}\text { Bloodstream, } \\
\text { shoulders }\end{array}$ & 8 \\
\hline 6 & $\begin{array}{l}\text { Intra-articular injection, } \\
\text { shoulder }\end{array}$ & Aug 22, 2018 & $\begin{array}{l}\text { Orthopedic } \\
\text { clinic }\end{array}$ & $\begin{array}{l}\text { Rotator cuff tear } \\
\text { with } \\
\text { intrasynovial cyst }\end{array}$ & Sep 9, 2018 & Escherichia coli & Shoulder & 6 \\
\hline 7 & $\begin{array}{l}\text { Intra-articular injection, } \\
\text { lumbar spine }\end{array}$ & Aug 28, 2018 & $\begin{array}{l}\text { Spine } \\
\text { treatment } \\
\text { clinic }\end{array}$ & Lumbar back pain & Sep 1, 2018 & Citrobacter koseri & Bloodstream & 6 \\
\hline 8 & $\begin{array}{l}\text { Intra-articular injection, } \\
\text { lumbar spine }\end{array}$ & Aug 29, 2018 & Pain clinic & Pain & Sep 4, 2018 & $\begin{array}{c}\text { Escherichia coli, } \\
\text { Enterococcus } \\
\text { faecalis }\end{array}$ & Bloodstream & 35 \\
\hline 9 & $\begin{array}{l}\text { Intra-articular injection, } \\
\text { knee }\end{array}$ & Aug 30, 2018 & $\begin{array}{l}\text { Orthopedic } \\
\text { clinic }\end{array}$ & Osteoarthritis & Sep 7, 2018 & $\begin{array}{c}\text { Escherichia coli, } \\
\text { Enterococcus } \\
\text { faecalis }\end{array}$ & Knee & 5 \\
\hline 10 & $\begin{array}{l}\text { Intra-articular injection, } \\
\text { cervical spine }\end{array}$ & Sep 12, 2018 & Pain clinic & Pain & Sep 15, 2018 & $\begin{array}{c}\text { Enterobacter } \\
\text { cloacae, } \\
\text { Citrobacter } \\
\text { freundii }\end{array}$ & $\begin{array}{l}\text { Bloodstream, } \\
\text { cellulitis at injection } \\
\text { site }^{\S}\end{array}$ & 9 \\
\hline 11 & $\begin{array}{l}\text { Intra-articular injection, } \\
\text { cervical and lumbar } \\
\text { spine }\end{array}$ & Sep 12, 2018 & Pain clinic & $\begin{array}{l}\text { Pain (history of } \\
\text { rheumatoid } \\
\text { arthritis) }\end{array}$ & Sep 16, 2018 & $\begin{array}{l}\text { Enterobacter } \\
\text { cloacae, } \\
\text { Citrobacter } \\
\text { freundii }\end{array}$ & Bloodstream & 12 \\
\hline 12 & $\begin{array}{l}\text { Intra-articular injection, } \\
\text { lumbar spine and index } \\
\text { fingers; intravenous } \\
\text { infusion }\end{array}$ & Sep 12, 2018 & Pain clinic & $\begin{array}{l}\text { Pain, rheumatoid } \\
\text { arthritis, } \\
\text { osteoarthritis }\end{array}$ & Sep 16, 2018 & $\begin{array}{c}\text { Enterobacter } \\
\text { cloacae }\end{array}$ & $\begin{array}{l}\text { Bloodstream, lumbar } \\
\text { epidural abscess }\end{array}$ & 12 \\
\hline
\end{tabular}

* As reported to CDC by health departments.

$\dagger$ Abscess and vertebrae were not cultured; both organisms were isolated from blood, and E. faecalis only was isolated from disc space.

$\S$ No organisms were isolated from skin; both organisms were isolated from blood.

\section{Acknowledgments}

Rachana Bhattarai, PhD, Kara Tarter, MPH, Arizona Department of Health Services; Robert Hunter, MS, Jon Rosenberg, MD, California Department of Public Health; Scott Pritchard, MPH, Virginia Warren, MPH, Bureau of Public Health Laboratories, Florida Department of Health; Texas Department of State Health Services; Ana Cecilia Bardossy, Gregory Eckert-Raczniak, MD, $\mathrm{PhD}$, Kathleen Hartnett, PhD, MD, Heather Moulton-Meissner, $\mathrm{PhD}, \mathrm{CDC}$.
Corresponding author: Kiran M. Perkins, KPerkins@cdc.gov, 404-639-1161.

${ }^{1}$ Division of Healthcare Quality Promotion, National Center for Emerging and Zoonotic Infectious Diseases, CDC; ${ }^{2}$ Florida Department of Health; ${ }^{3}$ Food and Drug Administration, Silver Spring, Maryland.

All authors have completed and submitted the ICMJE form for disclosure of potential conflicts of interest. No potential conflicts of interest were disclosed. 


\section{References}

1. Food and Drug Administration. FDA warns about stem cell therapies. Silver Spring, MD: Food and Drug Administration; 2017. https://www. fda.gov/ForConsumers/ConsumerUpdates/ucm286155.htm

2. Food and Drug Administration. Recall notification to clients with possible product on-hand, effective 9/28/18. Silver Spring, MD: Food and Drug Administration; 2018. https:/www.fda.gov/BiologicsBloodVaccines/ SafetyAvailability/Recalls/ucm622190.htm

3. Food and Drug Administration. Liveyon, LLC issues a voluntary nationwide recall of the Regen Series ${ }^{\oplus}$ product, manufactured by Genetech, Inc. Silver Spring, MD: Food and Drug Administration; 2018. https:// www.fda.gov/Safety/Recalls/ucm623039.htm
4. Food and Drug Administration. Guidance for industry: biologics license applications for minimally manipulated, unrelated allogeneic placental/umbilical cord blood intended for hematopoietic and immunologic reconstitution in patients with disorders affecting the hematopoietic system. Rockville, MD: Food and Drug Administration; 2014. https://www.fda.gov/downloads/ BiologicsBloodVaccines/GuidanceComplianceRegulatoryInformation/ Guidances/CellularandGeneTherapy/UCM357135.pdf

5. Marks PW, Witten CM, Califf RM. Clarifying stem-cell therapy's benefits and risks. N Engl J Med 2017;376:1007-9. https://doi.org/10.1056/ NEJMp1613723

6. Guh AY, Thompson ND, Schaefer MK, Patel PR, Perz JF. Patient notification for bloodborne pathogen testing due to unsafe injection practices in the US health care settings, 2001-2011. Med Care 2012;50:785-91. https://doi.org/10.1097/MLR.0b013e31825517d4 